\title{
Docetaxel-loaded lipid microbubbles combined with ultrasound-triggered microbubble destruction for targeted tumor therapy in MHCC-H cells
}

This article was published in the following Dove Press journal:

OncoTargets and Therapy

I August 2016

Number of times this article has been viewed

\author{
Yue Zhangl,* \\ Ruijiao Changl,* \\ Muqiong $\mathrm{Li}^{2}$,* \\ Kun Zhao ${ }^{3}$ \\ Hongzhi Zheng ${ }^{4}$ \\ Xiaodong Zhou' \\ 'Department of Ultrasound, Xijing \\ Hospital, ${ }^{2}$ Department of Chemistry, \\ School of Pharmacy, Fourth Military \\ Medical University, Xi'an, ${ }^{3}$ Department \\ of Cardiothoracic Surgery, The Third \\ Chinese People's Liberation Army \\ Hospital, Baoji, Shaanxi Province, \\ ${ }^{4}$ Department of Ultrasound, The \\ 534 Hospital, Luoyang, Henan \\ Province, People's Republic of China \\ *These authors contributed equally \\ to this work
}

Background: Efficient and targeted delivery of cytotoxic drugs is still a challenge in the fight against cancer. Ultrasound-targeted destruction of cytotoxic drug-loaded lipid microbubbles (LMs) might be a promising method. This study aimed to explore the antitumor effects of docetaxel-loaded LM (DLLM) combined with ultrasound-targeted microbubble destruction (UTMD) on liver cancer.

Materials and methods: DLLMs were made by a mechanical vibration technique. The effects of docetaxel, DLLM alone, and DLLM + UTMD on cell viability and cell proliferation (Cell Counting Kit- 8 assay) of MHCC-H cells and HepG2 cells were tested. The effects on cell cycle (flow cytometry) and apoptosis (flow cytometry and immunoblotting) of MHCC-H cells were tested. Solid fast-growing tumor mouse models were established and were randomized to blank LM + UTMD (controls) or DLLM + UTMD. Tumor volume was compared between the two groups. Results: DLLMs had an $18 \% \pm 7 \%$ drug-loading capacity, an $80 \% \pm 3 \%$ encapsulation efficiency, and a mean particle size of $2,845 \mathrm{~nm}$ (75\% range 1,527-5,534 nm). Compared to the other groups, DLLM + UTMD decreased the proliferation and increased the apoptosis of MHCC-H cells. DLLM + UTMD resulted in the inhibition of a higher proportion of cells in the G1 phase. Compared to the control group, the tumor volume in mice receiving DLLM + UTMD was smaller.

Conclusion: DLLM + UTMD can increase the proportion of cells arrested in the G1 phase, decrease tumor cell proliferation, and induce MHCC-H cell apoptosis. The growth of solid tumors in mice was inhibited. These results could provide a novel targeted strategy against liver cancer.

Keywords: lipid microbubble, liver cancer, docetaxel, ultrasound-triggered drug delivery

\section{Introduction}

Hepatocellular carcinoma (HCC) is a highly prevalent disease in the world. The prognosis of HCC is generally poor because of the low effectiveness of available treatments, resulting in an overall 5 -year survival of 5\%-6\%. ${ }^{1,2}$ Among the available treatments, resection, embolization, thermal ablation, and liver transplantation are used. There are many types of HCC, and some patients with $\mathrm{HCC}$ will not benefit from surgery because of vascular invasion, metastases, and poorly differentiated histology. ${ }^{3}$ To improve the prognosis, tumor response to therapy should be assessed as early as possible. ${ }^{4}$

Lipid microbubbles (LMs) have the ability to carry drugs into cells. ${ }^{5}$ Hydrophilic substances are encapsulated inside the LMs, while lipophilic drugs are mainly entrapped within the lipid bilayer. ${ }^{6}$ Various methods of liposome preparation have been described. ${ }^{7}$ LMs loaded with cytotoxic drugs have been developed and promising
Correspondence: Xiaodong Zhou Department of Ultrasound, Xijing Hospital, Fourth Military Medical University, 127 West of Changle Road, Xi'an 710032, Shaanxi Province, People's Republic of China

Tel +86 298477 I 355

Fax +86 2983244121

Email zhouxd@fmmu.edu.cn 
therapeutic effects have been obtained. ${ }^{8}$ Recently, ultrasoundtargeted microbubble destruction (UTMD) has emerged as a promising strategy for the efficient delivery of drugs that are poorly soluble in water., ${ }^{9,10}$ Ultrasound can be used to improve drug delivery to cancer cells via administration of drug-containing carriers. ${ }^{10,11}$ A number of previous studies have examined the efficacy of UTMD in $\mathrm{HCC}^{12}$ through the downregulation of CD133, ${ }^{13}$ gene delivery, ${ }^{14,15}$ cytotoxic drug delivery, ${ }^{16,17}$ and increased capillary permeability. ${ }^{18}$

Docetaxel (DOC) is a cytotoxic drug that is widely used in solid tumors (including HCC), ${ }^{19-22}$ but it is associated with systemic toxicities such as nausea, vomiting, diarrhea, febrile neutropenia, fatigue, fluid retention, pneumonitis, cutaneous and nail toxicity, epiphora and lacrimal duct stenosis, gastrointestinal complications, and neurotoxicity. ${ }^{23}$ Paclitaxel is rapidly cleared from the circulation after its intravenous administration, while DOC is not. ${ }^{24}$ Therefore, DOC-loaded LMs (DLLMs) might solve these problems. In previous studies by Unger et al, ${ }^{25}$ PEGylated liposomes containing DOC were successfully used in the treatment of liver metastases via hepatic arterial infusion.

As ultrasound contrast agents, microbubbles (MBs) have been used to carry drugs and genes, ${ }^{12,17}$ and the activation of MBs containing drugs using ultrasound can release the drugs in the target tissues. After intravenous injection, MBs can arrive at the target tissues and be destroyed by an ultrasound beam, and the consequent mechanical effects and cavitation could increase membrane permeability, causing rupture of microvessels (diameter $<7 \mu \mathrm{m}$ ) and widening of endothelial cell gaps, allowing the drugs to diffuse into the cells. ${ }^{26}$

Therefore, drug-loaded LMs used in combination with UTMD might provide a novel approach for targeted chemotherapy. ${ }^{27}$ The hypothesis of this study was that DLLMs combined with UTMD could be used to specifically target HCC. The aim of this study was to examine the antitumor efficacy of DLLM + UTMD in the MHCC-H cell line and in a mouse model of HCC.

\section{Materials and methods}

\section{Materials and instruments}

1,2-Dipalmitoyl-sn-glycero-3-phosphocholine and 1,2dipalmitoyl-sn-glycero-3-phosphoethanolamine- $N$ [methoxy(polyethylene glycol)-2000] (DPPE-PEG2000) were purchased from Corden Pharma International (Plankstadt, Germany). DOC (99.9\% purity) was purchased from Melone Pharmaceutical Co., Ltd. (Dalian, People's Republic of China). Methyl alcohol, ethyl alcohol (75\%), glycerin, normal saline, and $\mathrm{C}_{3} \mathrm{~F}_{8}$ gas were purchased from Dingguo Bio Co., (Xi'an, People's Republic of China). CD105 (Hoffman-La
Roche Ltd., Basel, Switzerland); Cell Counting Kit-8 (CCK-8; Dojindo Molecular Technologies, Kimamoto, Japan); TUNEL (Hoffman-La Roche Ltd.); 4',6-diamidino-2-phenylindole (Beijing Leagene Biotech Co., Ltd., Beijing, People's Republic of China); protease inhibitor (Beijing Leagene Biotech Co., Ltd.); serum without phenol red; fetal bovine serum (Beijing Leagene Biotech Co., Ltd.); and Dulbecco's Modified Eagle's Medium (DMEM; Thermo Fisher Scientific, Waltham, MA, USA) were used. Resistance of quenched tablets (Beyotime Institute of Biotechnology, Shanghai, People's Republic of China) was measured. Forma Series II Water-Jacketed $\mathrm{CO}_{2}$ Incubator (HEPA class 100; Thermo Fisher Scientific), Western blot primary antibody diluent (Xi' an Zhuangzhi Co., Xi'an, People's Republic of China), and Hg mixer (YaRong Co., Shanghai, People's Republic of China) were used. Also, coupling agent (Ambition T.C., Shenzhen, People's Republic of China), Caspase-3 Activity Assay Kit (Cell Signaling, Danvers, MA, USA), and cell lysis buffer (Beyotime Institute of Biotechnology, Shanghai, People's Republic of China) were used. The MHCC-H cell line was a kind gift from the Department of Hepatobiliary Surgery of the Xijing Hospital. They were maintained in DMEM-high glucose (HG) containing $10 \%$ fetal calf serum at $37^{\circ} \mathrm{C}$. The following apparatus were also used: glassware (Bejing Synthware Glass Co., Ltd., Beijing, People's Republic of China), laser scanning confocal microscope (Fluoview, FV10i, Model FV10C-W3; Olympus Corporation, Tokyo, Japan), transmission electron microscopy, flow cytometry (BD FACSAria, XL, EXPO32; Beckman Coulter, Brea, CA, USA), cell culture bottles (Corning Incorporated, Corning, NY, USA), ultrapure water system (Milli-Q, A10; EMD Millipore, Billerica, MA, USA), pH meter (PB-10; Sartorius, Goettingen, Germany), ultrasonic equipment and imaging system including a color/power and pulsed Doppler analysis (MyLab 90; Esaote S.p.A., Genova, Italy), small animal imaging (IVIS Lumina II; Caliper Life Sciences, Hopkinton, MA, USA), paraffin embedding station (YABO600 computer control; Yabo Electronic, Changzhou, Jiangsu, People's Republic of China), refrigerating machine (IMS-50; Jiangdong Instruments, Suzhou, Jiangsu, People's Republic of China), Thermo Scientific Multiskan FC (Thermo Fisher Scientific), Centrifuge (5424R; Eppendorf, Hamburg, Germany), and Fluoroskan (ChemiDoc XRS+; Bio-Rad Laboratories Inc., Hercules, CA, USA).

\section{Preparation of targeting DLLMs}

An appropriate amount of lipid mixture (1,2-Dipalmitoyl-snglycero-3-phosphocholine, DPPA, and biotin-DPPE-PEG2000 in a molar ratio of 47:10:5) and DOC with a mass ratio of $20 \%$ to total phospholipid was dissolved in chloroform and 
methanol $(1: 1, \mathrm{v}: \mathrm{v})$. The solution was transferred to a round bottom flask, and the solvent was removed by rotary vacuum evaporation at $60^{\circ} \mathrm{C}$. The resulting lipid film was hydrated in phosphate-buffered saline (PBS; $\mathrm{pH}$ 7.4). A proportional amount of glycerol $(9: 1, \mathrm{v}: \mathrm{v})$ was added to produce a final lipid concentration of $20 \mathrm{mg} / \mathrm{mL}$. The liposomal formulation underwent aqueous bathing at $60^{\circ} \mathrm{C}$ for 0.5 hours, and aliquots of $450 \mu \mathrm{L}$ in $1.5 \mathrm{~mL}$ were placed in round-bottom tubes. Perfluoropropane gas was bubbled using a syringe through the rubber caps. The dispersion was mechanically vibrated at 3,000 rpm for 45 seconds and then the DLLMs were obtained. They were washed with PBS three times and free drug (not incorporated into the MBs) was separated by centrifugal flotation. The unloaded MBs (nondrug loaded) were similarly prepared but without the addition of DOC. Both samples were observed and photographed using an inverted microscope (TH4-200; Olympus Corporation). The concentration and size of MBs were determined using a Delsa Nano analyzer (Beckman Coulter).

\section{Cell viability assay}

MHCC-H cells and HepG2 cells were seeded into 96-well plates at a density of $1 \times 10^{4}$ cells/well and grown in DMEM-HG containing $10 \%$ fetal bovine serum at $37^{\circ} \mathrm{C}$ for 48 hours. The cells were divided into four groups: control (CON), DOC only, DLLM, and DLLM + UTMD. Cell viability was evaluated by the CCK- 8 assay. In the DOC group, the cells were treated with DOC at $1 \mathrm{ng} / \mathrm{mL}$. The DLLM group was treated with the same amount of DLLMs. The DLLM + UTMD group was exposed to ultrasound 2 hours after the DLLMs were added. Optical density was measured at baseline, 1 hour, 2 hours, 4 hours, 8 hours, 24 hours, and 48 hours. ${ }^{28}$

\section{Cell cycle and apoptosis by flow cytometry} MHCC-H cells were seeded into 12 -well plates at a density of $1 \times 10^{6}$ cells/well and grown in DMEM-HG containing $10 \%$ fetal bovine serum at $37^{\circ} \mathrm{C} .{ }^{29}$ The cells were treated in the CON, DOC only, DLLM, and DLLM + UTMD groups for 48 hours. Then, MHCC-H cells were digested with PBS and centrifuged at 1,000 rpm for 5 minutes. Samples for apoptosis testing were added to $2.5 \mathrm{~mL}$ of serum culture medium. Cells for cell cycle testing were added to $1 \mathrm{~mL}$ of PBS, fixed with $2 \mathrm{~mL}$ of absolute ethyl alcohol, and kept at $4{ }^{\circ} \mathrm{C}$.

\section{Immunoblotting}

MHCC-H cells were collected and quantified by BCA Protein Quantitation Kit. Briefly, the proteins were separated by sodium dodecyl sulfate polyacrylamide gel electrophoresis on $16 \%(\mathrm{w} / \mathrm{v})$ polyacrylamide gels, transferred onto polyvinylidene difluoride membranes (EMD Millipore), and then blocked in Tris-buffered saline containing $0.05 \%$ Tween 20 and 5\% nonfat dry milk for 24 hours overnight. The blots were then incubated with cleaved caspase- 3 antibody or $\beta$-actin antibody for 1.5 hours at room temperature. The blots were examined using a ChemiDoc ${ }^{\mathrm{TM}} \mathrm{XRS}+$ System (Bio-Rad Laboratories Inc.) and quantified using the software ImagePlus.

\section{Animal models}

Six-week-old male nude mice (25-27 g) were supplied by the Laboratory Animal Center of the Fourth Military Medical University and maintained according to guidelines from the local animal care committee. MHCC-H cells in the logarithmic growth phase were collected, washed thrice in PBS, and inoculated subcutaneously into the dorsal flank area of the mice $\left(5 \times 10^{6}\right.$ cells in $100 \mu \mathrm{L}$ of PBS per mouse). Tumors were allowed to grow until they reached $\sim 5 \mathrm{~mm}$ at the tenth day after inoculation. All animal experiments were performed with permission from the Animal Ethical Commission of the Fourth Military Medical University.

Ultrasound imaging was performed using a MyLab 90 LA332 linear transducer. Unloaded LMs were used as CON. Tumor-bearing mice received a bolus injection of $0.2 \mathrm{~mL}$ of DLLMs through the tail vein. Tumors were continuously monitored for 20 minutes with contrast-enhanced ultrasonography.

After 10 days, ten mice were randomized: the CON group was treated with unloaded LMs + UTMD and the experimental group was treated with DLLMs + UTMD (once a day for ten consecutive days). Each mouse received the same volume of bolus injection $(0.2 \mathrm{~mL})$ through the tail vein. The same volume of DLLMs or DOC was administrated in the experimental groups. Ultrasound exposure (mechanical index [MI] 0.4, power [P] 80\%) was conducted using a general ultrasound transducer (MyLab 90 LA332) using a nonfocused probe, as previously described. ${ }^{27}$

The tumors were measured using a Vernier caliper once a day until the eleventh day of treatment. Tumor volume $(V)$ was calculated according to $V=\left(L \times S^{2}\right) / 2$, where $L$ and $S$ are the longest and shortest dimensions of the tumor in millimeter, respectively.

\section{Statistical analysis}

All data are presented as mean \pm standard deviation and were analyzed using one-way analysis of variance with the post hoc $t$-test. Statistical analysis was carried out using SPSS 15.0 
(SPSS Inc., Chicago, IL, USA). Two-sided $P$-values $<0.05$ were considered significant.

\section{Results}

\section{Characteristics of the DLLMs}

Under electron microscopy, the DLLMs had a round shape and most of them were separated from each other (Figure 1A and $\mathrm{B}, 50,000 \times$ and 10,000×, respectively). DLLMs had an $18 \% \pm 7 \%$ drug-loading capacity, an $80 \% \pm 3 \%$ encapsulation efficiency, and a mean particle size of $2,845 \mathrm{~nm}$ (75\% range 1,527-5,534 nm) (Figure 1C). The size distribution was Gaussian. The DLLMs were stable for 10 days at $4^{\circ} \mathrm{C}$. The zeta potential was $2.39 \mathrm{mV}$.

\section{DLLM + UTMD inhibited MHCC-H and HepG2 cell viability and proliferation}

Cell viability and proliferation tests were performed to directly reflect the antitumor effect of DLLM. In the first 2 hours, cell viability in the DOC group was significantly lower than that in the other groups. While the viability of the DLLM group and DLLM + UTMD group decreased in a time-dependent manner, during the 4 to 48 hour period after UTMD, the DLLM + UTMD group had the lowest cell viability compared to the other groups (Figure 2A and B). Cell proliferation in the DLLM + UTMD group after 24 hours, 48 hours, and 72 hours was lower compared to the three other groups (Figure 2C and D).

\section{DLLM + UTMD inhibited MHCC-H cell cycle}

Cell cycle analysis showed a significantly lower proportion of cells in the G2 + M phase in the DLLM + UTMD group, which suggested that in the DLLM + UTMD group, the cell cycle was inhibited in the G1 phase compared to the three other treatment groups (Figure $3 \mathrm{~A}-\mathrm{C}$ ).

\section{DLLM + UTMD stimulated MHCC-H apoptosis}

Flow cytometry analysis showed that DLLM + UTMD promoted apoptosis in MHCC-H cells compared to the three other groups (Figure 4A and B). Furthermore, to evaluate the activation of caspase- 3 , the amount of cleaved caspase- 3 was
A

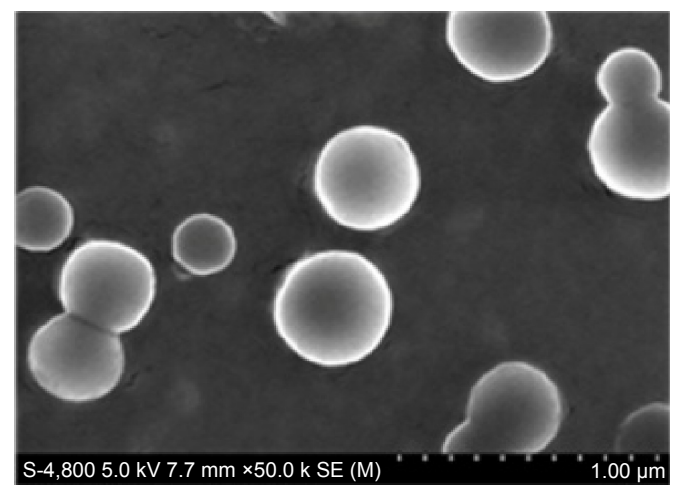

B

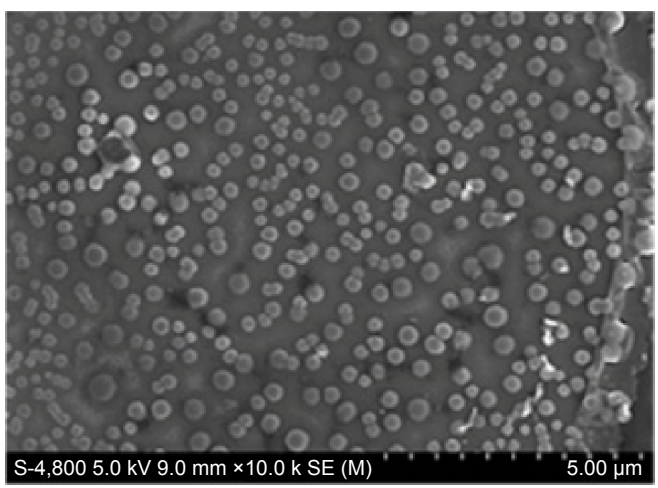

C

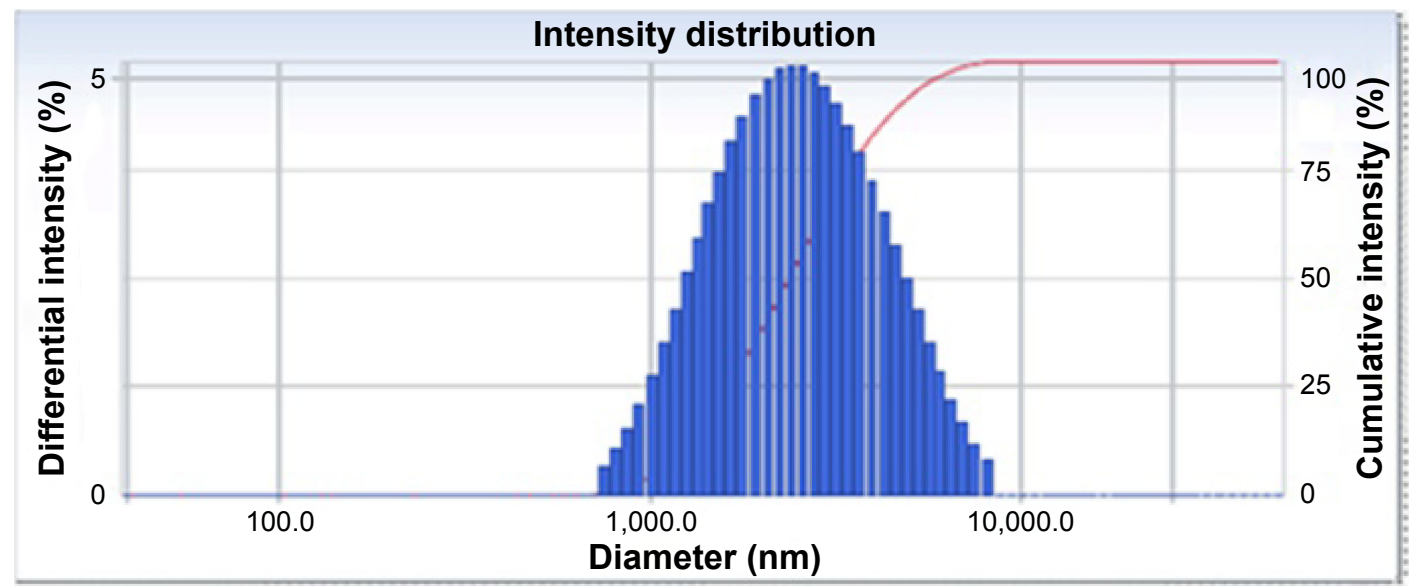

Figure I Morphology of the DLLMs under electron microscopy and particle size analysis.

Notes: (A) Scanning electron microscopy at 50,000x. (B) Scanning electron microscopy at 10,000x. (C) Average particle size tested by laser particle size analysis. Abbreviation: DLLMs, docetaxel-loaded lipid microbubbles. 
A

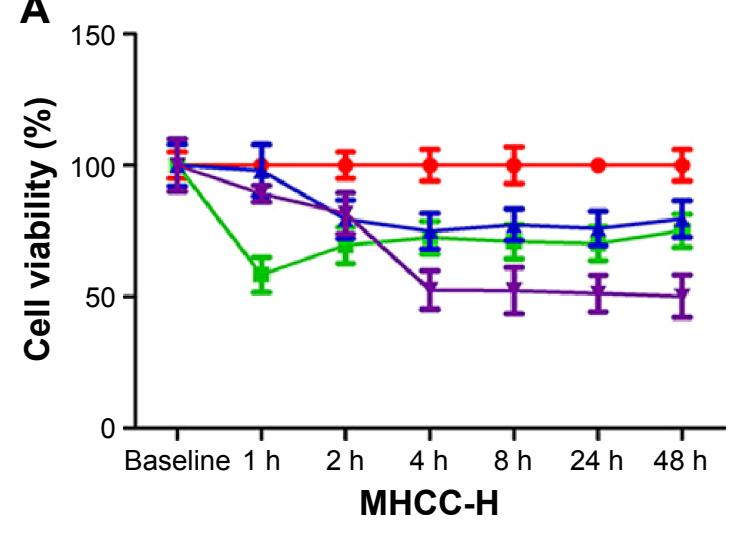

B

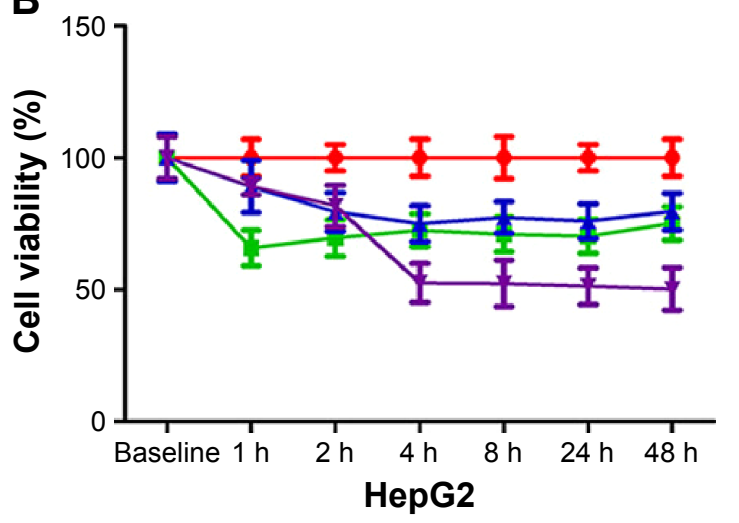

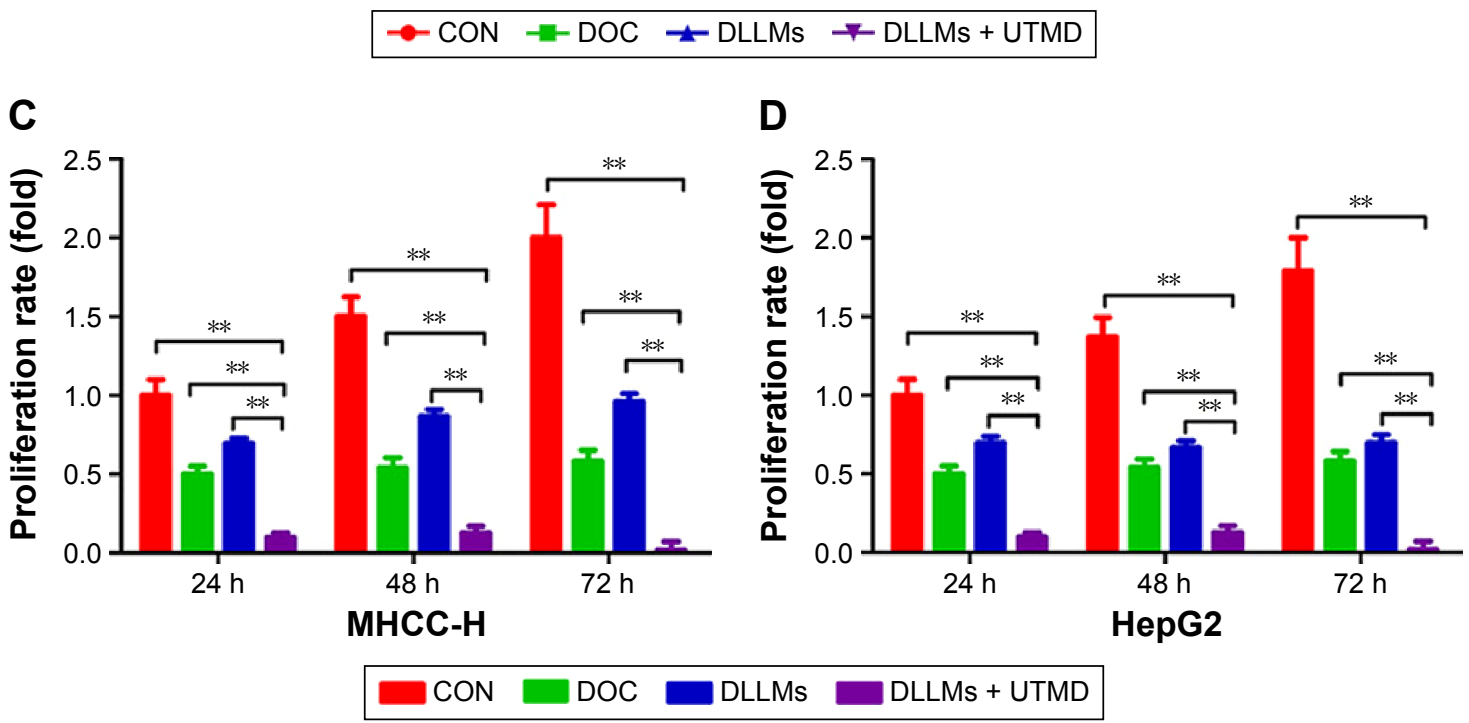

Figure 2 CCK-8 cell viability assay of MHCC-H cells and HepG2 cells.

Notes: MHCC-H cells ( $\mathbf{A}$ and $\mathbf{C}$ ) and HepG2 cells (B and $\mathbf{D})$ treated with CON, DOC, DLLMs, and DLLMs + UTMD were harvested at the indicated time ( $\mathrm{n}=4 /$ group). (A and B) CCK-8 cell viability assay of MHCC-H cells and HepG2 cells at 0-48 h. (C and D) The proliferation ratio (fold) assay of MHCC-H cells and HepG2 cells at $24 \mathrm{~h}$, $48 \mathrm{~h}$, and $72 \mathrm{~h}$ from three independent experiments. Mean \pm standard deviation is shown. $* * P<0.0 \mathrm{I}$.

Abbreviations: CCK-8, Cell Counting Kit-8; CON, control; DOC, docetaxel; DLLMs, docetaxel-loaded lipid microbubbles; UTMD, ultrasound-targeted microbubble destruction; h, hours.

determined by immunoblotting. In accordance with earlier experiments, the quantity of cleaved caspase- 3 protein level was significantly higher in the DLLM + UTMD group than the other groups (Figure 4C).

\section{DLLM + UTMD suppressed tumor growth in a mouse model}

After 10 days of treatment, the tumor volume in the DLLM + UTMD group was smaller compared to the CON group (Figure 5A). In addition, the tumor size was significantly decreased in the DLLM + UTMD group compared to the CON group (Figure 5B).

\section{Discussion}

The aim of this study was to explore the antitumor effects of DLLMs combined with UTMD on HCC. The results showed that DLLM had an $18 \% \pm 7 \%$ drug-loading capacity, $80 \% \pm 3 \%$ encapsulation efficiency, and mean particle size of $2,845 \mathrm{~nm}$ (75\% range 1,527-5,534 nm). DLLM+UTMD decreased proliferation, increased apoptosis in MHCC-H cells, and resulted in a higher proportion of cells arrested in the G1 phase. DLLM was successfully linked to CD105. DLLM-CD105 had targeting ability against MHCC-H cells loaded in nude mice. Compared to the CON group, the tumor volume in the DLLM + UTMD group was smaller.

Previous studies have explored the effects of drug-delivering LMs in HCC. Liu et $\mathrm{al}^{30}$ have shown that DOC-loaded nanoparticles had the property of entering into the tumors, improving the targeted delivery of DOC. Similar results have been obtained using paclitaxel. ${ }^{31}$ Another study by the same group showed that UTMD using particles loaded with short hairpin RNA against CD133 was efficient at preventing the 
A
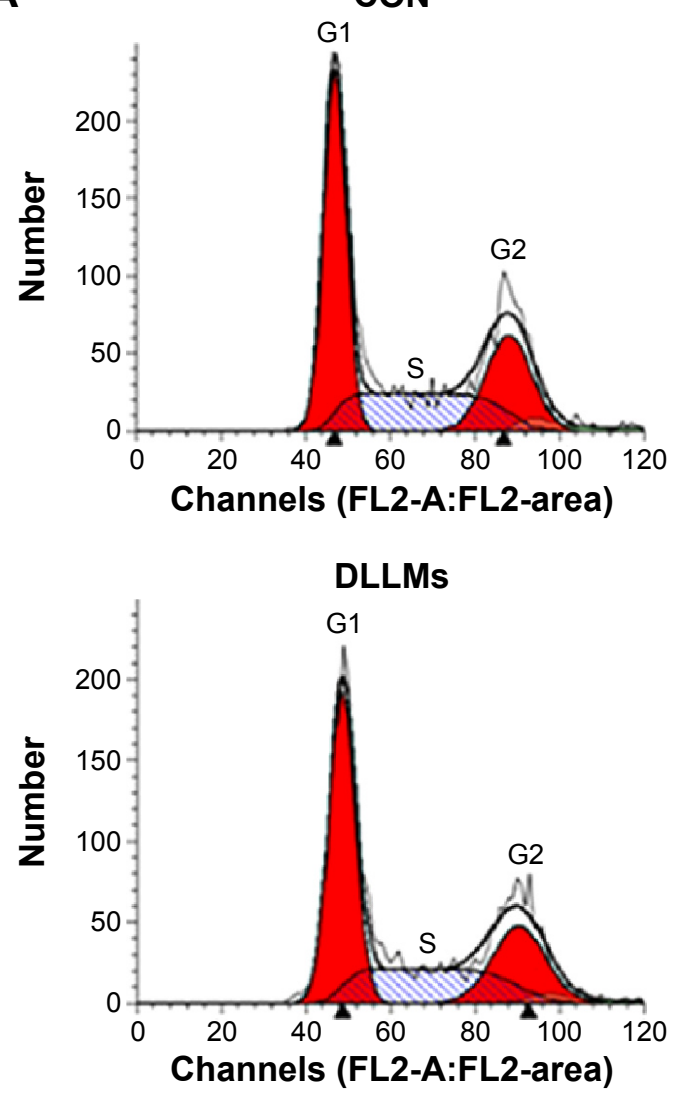

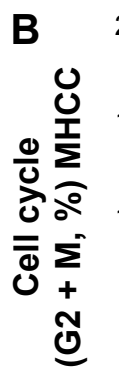

CON
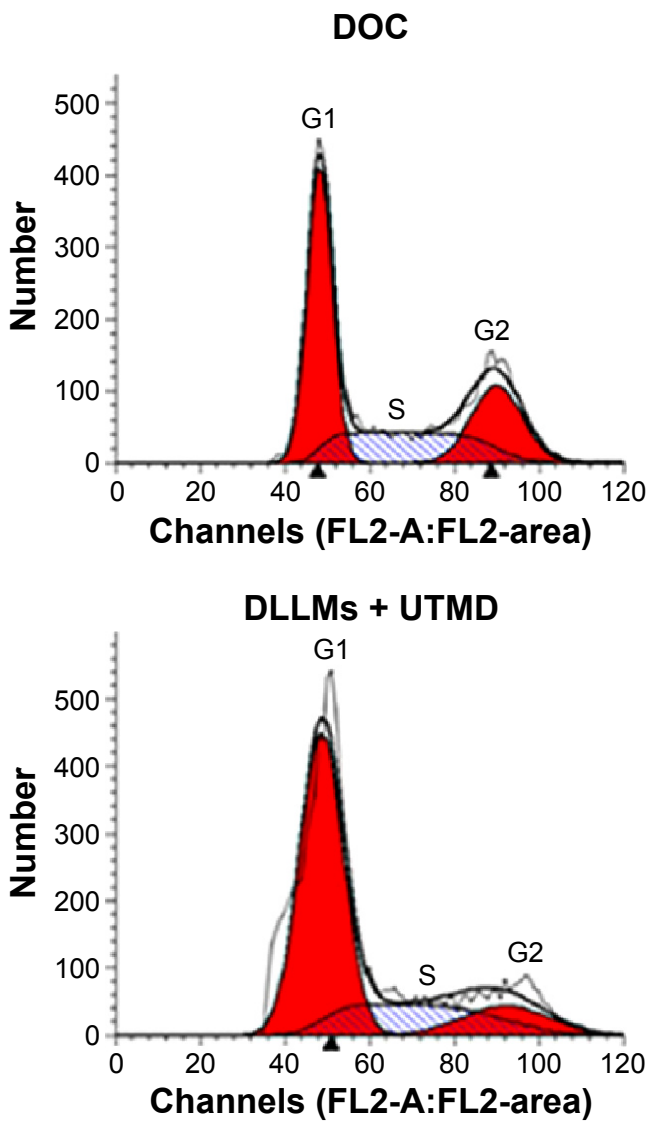
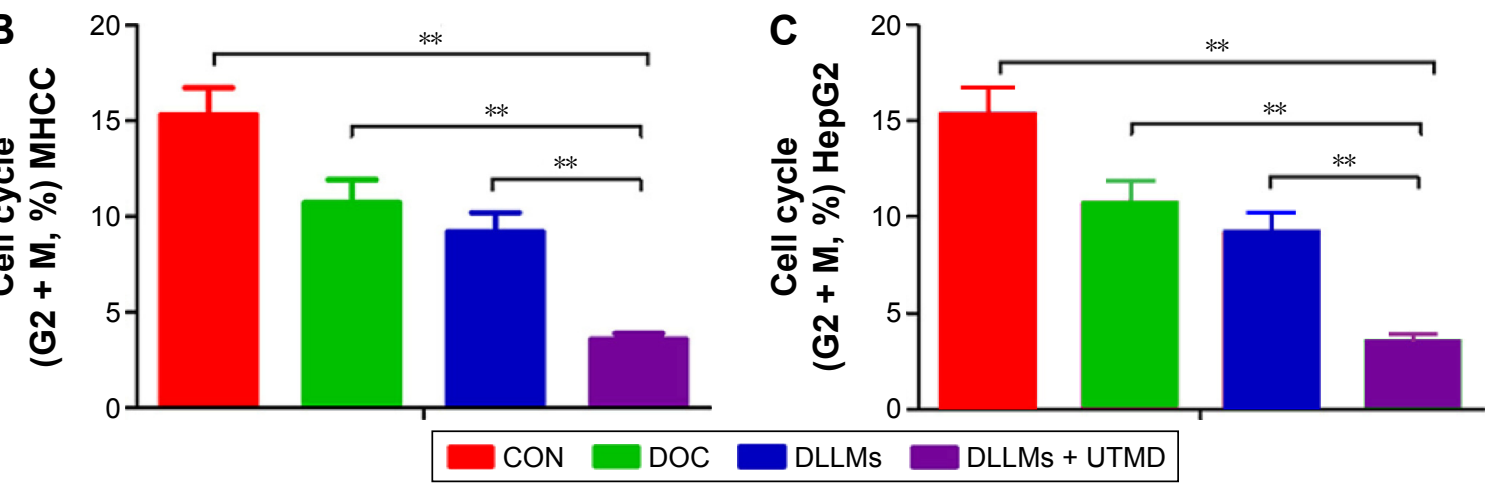

Figure 3 Flow cytometric analysis of cell cycles of MHCC-H cells.

Notes: (A) MHCC-H cells treated with CON, DOC, DLLMs, and DLLMs + UTMD for $48 \mathrm{~h}$ were harvested ( $\mathrm{n}=6 / \mathrm{group})$. The DLLMs + UTMD treatment increased the number of MHCC-H cells arrested in the GI phase. (B) Quantitative analysis of MHCC-H cells from three independent experiments. Mean $\pm s t a n d a r d ~ d e v i a t i o n$ is shown. **P $<0.0 \mathrm{I}$. (C) Quantitative analysis of Hep-G2 cells from three independent experiments. Mean \pm standard deviation is shown. $* * P<0.0$ I.

Abbreviations: CON, control; DOC, docetaxel; DLLMs, docetaxel-loaded lipid microbubbles; UTMD, ultrasound-targeted microbubble destruction; h, hours.

epithelial-mesenchymal transition of HCC cells. ${ }^{13}$ A similar UTMD strategy was used to deliver a suicide gene into HCC cells and showed that the UTMD approach was specific to the tissues being targeted by ultrasound. ${ }^{15}$ In addition, ultrasounds themselves have been shown to improve the accumulation of nanoparticles in tumors, potentially leading to a synergistic effect against tumors. ${ }^{22}$ Ultrasounds also break capillaries, increasing the vessel permeability in targeted areas. ${ }^{18}$

In this study, the use of DLLM + UTMD significantly led to inhibition of cell proliferation, inhibition of cell cycle in G1 phase, and inhibition of apoptosis in HCC cells. In addition, in vivo, this treatment strategy resulted in the inhibition of growth of HCC tumors, which is consistent with a previous study.$^{16}$ In this study, except the lack of toxicities, the effects of DOC on the tumors were consistent with the mechanisms of action of DOC. Indeed, DOC is known to stabilize the microtubules and prevent the cells from entering mitosis, triggering apoptosis. In this study, DLLM + UTMD had exactly this effect in vitro, and we can hypothesize that it had the same effect in vivo. On the other hand, some 


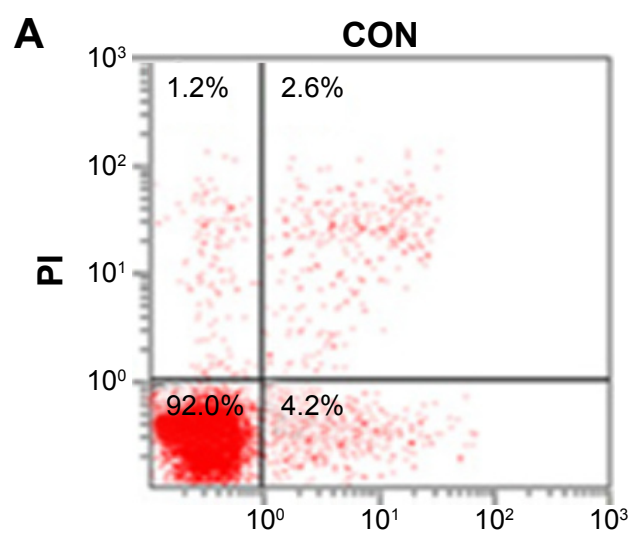

Annexin V-FITC

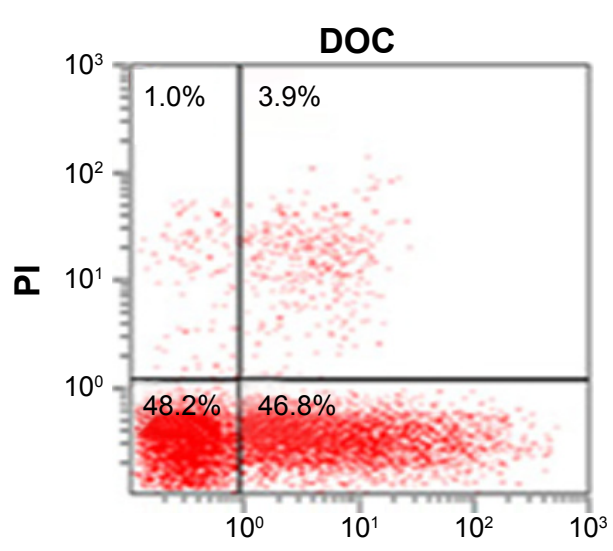

Annexin V-FITC

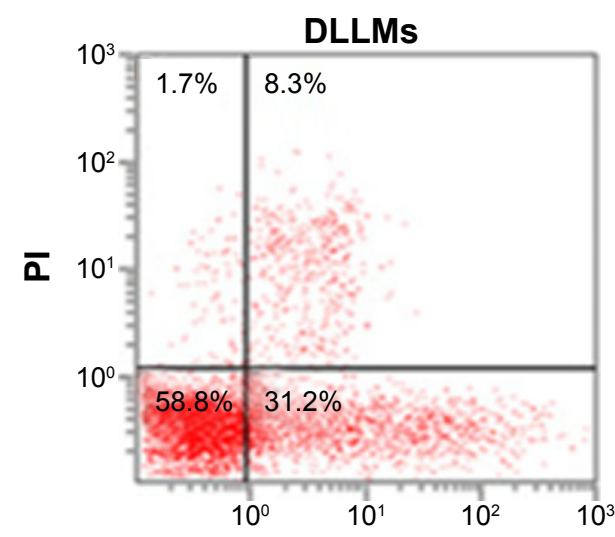

Annexin V-FITC

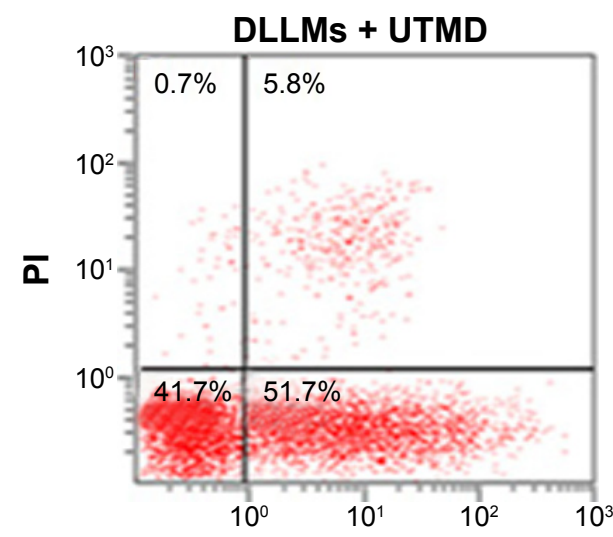

Annexin V-FITC
B
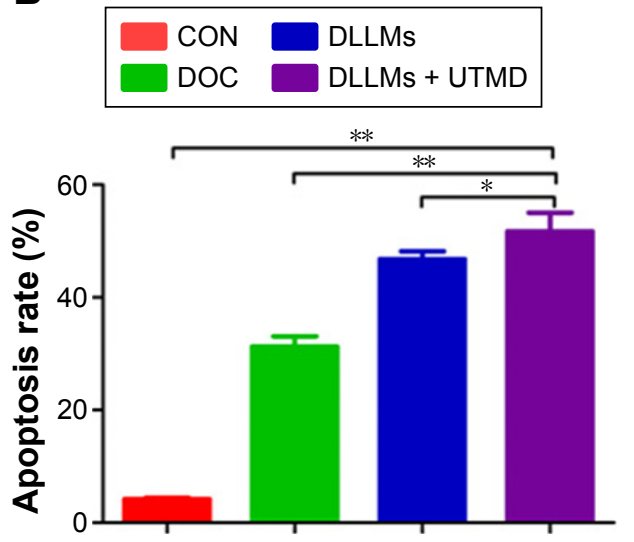

C

Cleaved caspase-3
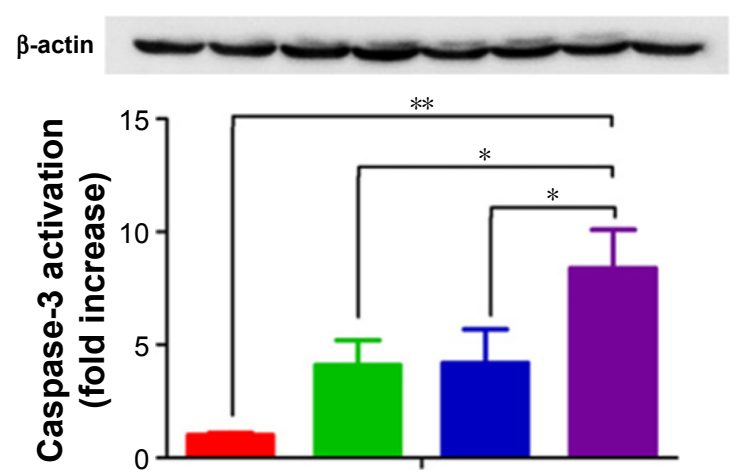

Figure 4 Flow cytometric analysis of apoptosis of MHCC-H cells.

Notes: MHCC-H cells treated with CON, DOC, DLLMs, and DLLMs + UTMD for $48 \mathrm{~h}$ were harvested ( $\mathrm{n}=6 / \mathrm{group}$ ). (A) Apoptosis assay by flow cytometric analysis. (B) Quantification of apoptosis. (C) Western blot assay of cleaved caspase-3 and quantification from three independent experiments. Mean \pm standard deviation is shown. $* P<0.05$ and $* * P<0.01$.

Abbreviations: CON, control; DOC, docetaxel; DLLMs, docetaxel-loaded lipid microbubbles; UTMD, ultrasound-targeted microbubble destruction; h, hours; FITC, fluorescein isothiocyanate.

previous studies suggested that the antitumor mechanism of DOC also includes inhibition of angiogenesis. ${ }^{32-35}$ However, additional studies are necessary to evaluate the exact contribution if these mechanisms in the treatment of HCC by DLLM + UTMD and the possibility of other mechanisms being involved.
Though the effect of DLLM + UTMD treatment on HCC was proved in MHCC-H cells and mice, there is a long way to go before the first clinical trial. The dose used in this work did not cause any adverse effect. However, the toxicities of the DLLM + UTMD treatment need to be studied in the future. Otherwise, studies about the pharmacokinetics of 


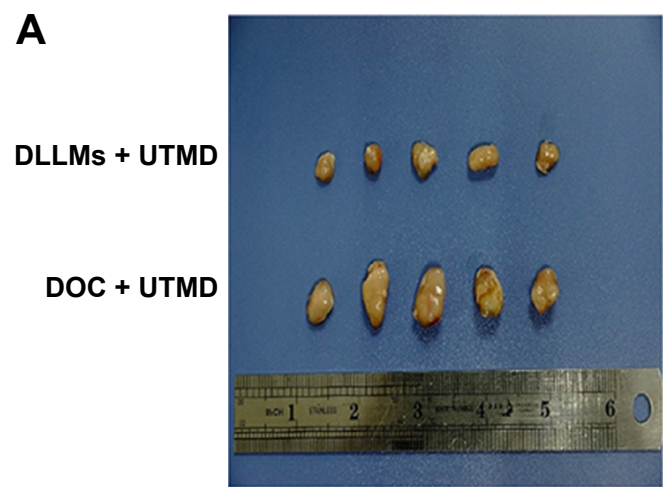

\section{B}

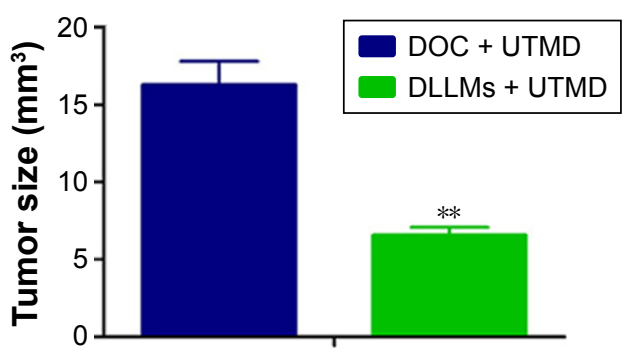

Figure 5 Effects of DLLMs + UTMD on MHCC-H xenografts.

Notes: Tumor growth was inhibited by DLLMs + UTMD in a mouse model. (A) The tumor volume of the DLLMs + UTMD group (upper) was much smaller compared to the CON group (lower). (B) Quantification of the tumor size in both groups ( $n=5 /$ group). ${ }^{* *} \mathrm{P}<0.01$ vs CONs.

Abbreviations: DLLMs, docetaxel-loaded lipid microbubbles; UTMD, ultrasound-targeted microbubble destruction; CON, control; DOC, docetaxel.

DLLM + UTMD will be helpful to select the best dose for future clinical trials. Therefore, more detailed studies are necessary to address these issues.

\section{Conclusion}

DLLM + UTMD could increase the proportion of cells arrested in G1 phase, decrease tumor cell proliferation, and induce apoptosis in MHCC-H cells. DLLM + UTMD significantly inhibited the growth of solid tumors in mice. These results suggested that DLLM + UTMD could be a novel potential targeted strategy against HCC.

\section{Acknowledgment}

This study was supported by grants from the National Natural Science Foundation of China (No 81071169 and No 81371567).

\section{Disclosure}

The authors report no conflicts of interest in this work.

\section{References}

1. Davila JA. Diabetes and hepatocellular carcinoma: what role does diabetes have in the presence of other known risk factors? Am J Gastroenterol. 2010;105(3):632-634.

2. Thomas MB, Zhu AX. Hepatocellular carcinoma: the need for progress. J Clin Oncol. 2005;23(13):2892-2899.

3. Shimada M, Rikimaru T, Hamatsu T, et al. The role of macroscopic classification in nodular-type hepatocellular carcinoma. Am J Surg. 2001; 182(2):177-182.

4. Jiang T, Zhu AX, Sahani DV. Established and novel imaging biomarkers for assessing response to therapy in hepatocellular carcinoma. J Hepatol. 2013;58(1):169-177.

5. Gourevich D, Hertzberg Y, Volovick A, et al. Ultrasound-mediated targeted drug delivery generated by multifocal beam patterns: an in vitro study. Ultrasound Med Biol. 2013;39(3):507-514.

6. Cui J, Gong Z, Shen HM. The role of autophagy in liver cancer: molecular mechanisms and potential therapeutic targets. Biochim Biophys Acta. 2013;1836(1):15-26.
7. Westwood M, Joore M, Grutters J, et al. Contrast-enhanced ultrasound using SonoVue(R) (sulphur hexafluoride microbubbles) compared with contrast-enhanced computed tomography and contrast-enhanced magnetic resonance imaging for the characterisation of focal liver lesions and detection of liver metastases: a systematic review and costeffectiveness analysis. Health Technol Assess. 2013;17(16):1-243.

8. Kang J, Wu X, Wang Z, et al. Antitumor effect of docetaxel-loaded lipid microbubbles combined with ultrasound-targeted microbubble activation on VX2 rabbit liver tumors. J Ultrasound Med. 2010;29(1):61-70.

9. Li P, Zheng Y, Ran H, et al. Ultrasound triggered drug release from 10-hydroxycamptothecin-loaded phospholipid microbubbles for targeted tumor therapy in mice. J Control Release. 2012;162(2):349-354.

10. Evjen TJ, Hupfeld S, Barnert S, Fossheim S, Schubert R, Brandl M. Physicochemical characterization of liposomes after ultrasound exposure - mechanisms of drug release. J Pharm Biomed Anal. 2013; 78-79:118-122.

11. Ren ST, Liao YR, Kang XN, et al. The antitumor effect of a new docetaxel-loaded microbubble combined with low-frequency ultrasound in vitro: preparation and parameter analysis. Pharm Res. 2013; 30(6):1574-1585.

12. Jain K, Kesharwani P, Gupta U, Jain NK. A review of glycosylated carriers for drug delivery. Biomaterials. 2012;33(16):4166-4186.

13. Liu YM, Li XF, Liu H, Wu XL. Ultrasound-targeted microbubble destruction-mediated downregulation of CD133 inhibits epithelialmesenchymal transition, stemness and migratory ability of liver cancer stem cells. Oncol Rep. 2015;34(6):2977-2986.

14. Jing H, Cheng W, Zhang JW, Han X, Shao H, Sun YX. Galactosylated poly-L-lysine targeted microbubbles for ultrasound mediated antisense c-myc gene transfection in hepatocellular carcinoma cells. Arch Med Sci. 2015;11(2):292-300.

15. Zhou S, Li S, Liu Z, et al. Ultrasound-targeted microbubble destruction mediated herpes simplex virus-thymidine kinase gene treats hepatoma in mice. J Exp Clin Cancer Res. 2010;29:170.

16. Zhu F, Jiang Y, Luo F, Li P. Effectiveness of localized ultrasoundtargeted microbubble destruction with doxorubicin liposomes in H22 mouse hepatocellular carcinoma model. J Drug Target. 2015; 23(4):323-334.

17. Kooiman K, Vos HJ, Versluis M, de Jong N. Acoustic behavior of microbubbles and implications for drug delivery. Adv Drug Deliv Rev. 2014;72:28-48.

18. Bekeredjian R, Kroll RD, Fein E, et al. Ultrasound targeted microbubble destruction increases capillary permeability in hepatomas. Ultrasound Med Biol. 2007;33(10):1592-1598.

19. Chen F, Zhang J, Wang L, Wang Y, Chen M. Tumor pHe-triggered charge-reversal and redox-responsive nanoparticles for docetaxel delivery in hepatocellular carcinoma treatment. Nanoscale. 2015;7(38): 15763-15779. 
20. Li Q, Zhu LZ, Yang RJ, Zhu X. Cytotoxic activity of anticancer drugs on hepatocellular carcinoma cells in hypoxic-hyponutritional culture. Int Surg. 2014;99(6):745-752.

21. Jin Y, Cao B, Zhang M, et al. RASSF10 suppresses hepatocellular carcinoma growth by activating P53 signaling and methylation of RASSF10 is a docetaxel resistant marker. Genes Cancer. 2015;6(5-6):231-240.

22. Wei H, Huang J, Yang J, et al. Ultrasound exposure improves the targeted therapy effects of galactosylated docetaxel nanoparticles on hepatocellular carcinoma xenografts. PLoS One. 2013;8(3):e58133.

23. Bonneterre J, Spielman M, Guastalla JP, et al. Efficacy and safety of docetaxel (Taxotere) in heavily pretreated advanced breast cancer patients: the French compassionate use programme experience. Eur J Cancer. 1999;35(10):1431-1439.

24. Feng L, Mumper RJ. A critical review of lipid-based nanoparticles for taxane delivery. Cancer Lett. 2013;334(2):157-175.

25. Unger EC, Porter T, Culp W, Labell R, Matsunaga T, Zutshi R. Therapeutic applications of lipid-coated microbubbles. Adv Drug Deliv Rev. 2004;56(9):1291-1314.

26. Anselmo AC, Mitragotri S. An overview of clinical and commercial impact of drug delivery systems. J Control Release. 2014;190:15-28.

27. Wu W, Cheng Y, Guo BH, Wu Q. Pharmacokinetics of liver-targeted docetaxel liposomes modified with 6-O-acyl-D-galactose esters in rabbits. Biomed Rep. 2014;2(4):545-548.

28. Maaser K, Hopfner M, Kap H, et al. Extracellular nucleotides inhibit growth of human oesophageal cancer cells via P2Y(2)-receptors. Br J Cancer. 2002;86(4):636-644.
29. Vindeløv L, Christensen IJ. An integrated set of methods for routine flow cytometric DNA analysis. Methods Cell Biol. 1990;33:127-137.

30. Liu Q, Li R, Zhu Z, et al. Enhanced antitumor efficacy, biodistribution and penetration of docetaxel-loaded biodegradable nanoparticles. Int J Pharm. 2012;430(1-2):350-358.

31. Zhou Q, Ching AK, Leung WK, et al. Novel therapeutic potential in targeting microtubules by nanoparticle albumin-bound paclitaxel in hepatocellular carcinoma. Int J Oncol. 2011;38(3):721-731.

32. Sasaki H, Klotz LH, Sugar LM, Kiss A, Venkateswaran V. A combination of desmopressin and docetaxel inhibit cell proliferation and invasion mediated by urokinase-type plasminogen activator (uPA) in human prostate cancer cells. Biochem Biophys Res Commun. 2015; 464(3):848-854.

33. Borghese C, Cattaruzza L, Pivetta E, et al. Gefitinib inhibits the crosstalk between mesenchymal stem cells and prostate cancer cells leading to tumor cell proliferation and inhibition of docetaxel activity. $J$ Cell Biochem. 2013;114(5):1135-1144.

34. Gao L, Cui Y, He Q, Yang Y, Fei J, Li J. Selective recognition of co-assembled thrombin aptamer and docetaxel on mesoporous silica nanoparticles against tumor cell proliferation. Chemistry. 2011; 17(47):13170-13174.

35. Miyoshi Y, Kurosumi M, Kurebayashi J, et al; Collaborative Study Group of Scientific Research of the Japanese Breast Cancer Society. Low nuclear grade but not cell proliferation predictive of pathological complete response to docetaxel in human breast cancers. J Cancer Res Clin Oncol. 2008;134(5):561-567.
OncoTargets and Therapy

\section{Publish your work in this journal}

OncoTargets and Therapy is an international, peer-reviewed, open access journal focusing on the pathological basis of all cancers, potential targets for therapy and treatment protocols employed to improve the management of cancer patients. The journal also focuses on the impact of management programs and new therapeutic agents and protocols on

\section{Dovepress}

patient perspectives such as quality of life, adherence and satisfaction. The manuscript management system is completely online and includes a very quick and fair peer-review system, which is all easy to use. Visit http://www.dovepress.com/testimonials.php to read real quotes from published authors. 